\title{
Personalised or stratified medicine
}

\author{
Per Hartvig Honoré
}

Personalised medicine may never become a reality. Given all the complex processes that drugs undergo in the body, determining the pharmacokinetics and pharmacodynamics in detail will never be possible to understand and encounter. Drug doses are arrived at by many methods, most of which are empirical. Others are more sophisticated, such as dosing based on measurable signs or adverse effects. The use of biomarkers showing disease activity or adverse effects from imaging is at the forefront of developments to achieve more individual dosing. Dosing for individual patients by a combination of pharmacokinetics and pharmacodynamics is still a research focus, and some studies have reached the clinic. In spite of this, the overall impact is mainly to adjust doses to avoid unwanted effects in an individual.

Population-based pharmacokinetic and pharmacodynamic methods have become valued tools to improve dosing, particularly for cancer drugs which usually have a large interindividual variability in response and outcome. Populationbased pharmacokinetic studies enable the identification of the characteristics of a patient population which significantly influence the pharmacokinetic parameters but also take into account variability in pharmacodynamics. In general, populationbased kinetic studies do not focus on the individual patient as the unit of analysis. By doing so, limited data accumulated

Correspondence to Professor Per Hartvig Honoré, Department of Drug Design and Pharmacology, SUND, University of Copenhagen, 2100 Copenhagen, Denmark; peh@sund.ku.dk from many individuals can be analysed and a more representative sample of the target population is obtained. It is possible to describe the mean tendencies of the population (ie, the typical values) and also to describe the random effects including variability between subjects, between episodes and within a subject (residual variability). In this way, models can be constructed where correlations between the relevant patient population characteristics and pharmacokinetic parameters are described for an entire group of people. Such population models can then be used to predict the optimal dosage for individual patients based on the values of the given characteristics for that individual. This is the ultimate aim of individualising dosing and is known as Bayesian forecasting.

An increasing number of studies are now available which demonstrate the usefulness of population pharmacokinetic modelling. The majority of these are for cytotoxic drugs, but only to predict and avoid adverse effects rather than to foresee outcome. The reason is that the pharmacokinetics of the drug can be adequately described and its dependence on statistically significant patient variables is also known in order to tailor doses. The relationship between the pharmacokinetics and pharmacodynamics might also be elucidated with respect to adverse effects as occurring in different tissues of the patient Nevertheless, there is a third important player in cancer-that is, the tumour. The pharmacokinetics in most tumours are totally different from patient characteristics and depend on tumour blood flow and perfusion, transporters present for influx or efflux of the drug, internal metabolism or drug capturing. We know little about these mechanisms and quantifying their variance is still not possible. The same is true for the effects of drugs on cancer cells, which may be of different types, housing different targets with different binding properties. This knowledge is generally lacking with respect to tumour biology and effect. Indeed, cancer drugs might be dosed by weight, by surface area, by dose banding and even in fixed doses with similar results with respect to the effect on the tumour. Some studies surprisingly conclude that fixed dosing is a good alternative for treatment.

In fact these new methods cannot be described as personalised dosing, merely stratified dosing. Some characteristics of the patient can, in the best case, be identified as a cofactor of statistical significance that will influence dosing. Such significant cofactors might be age and/or kidney function which can be determined quantitatively and give an exact measure for decreasing a dose. However, random errors are still inherently present with these methods due to analysis precision, non-linearity and minor patientrelated cofactors not reaching statistical significance with respect to drug metabolism and targeting. The dose is just stratified with respect to some variables, but not all. The methods are still in their infancy, are mostly research tools and few have been able to isolate more than a small number of factors that should be considered in quantifying the magnitude of an altered dose.

Competing interests None.

Provenance and peer review Commissioned; internally peer reviewed.

European Journal of Hospital Pharmacy

2012;19:497.

doi:10.1136/ejhpharm-2012-000246 